\title{
CREB5 promotes tumor cell invasion and correlates with poor prognosis in epithelial ovarian cancer
}

\author{
SHANYANG HE* , YALAN DENG* ${ }^{*}$, YANBIN LIAO, XIAOJIN LI, JUN LIU and SHUZHONG YAO \\ Department of Obstetrics and Gynecology, The First Affiliated Hospital, \\ Sun Yat-sen University, Guangzhou, Guangdong 510700, P.R. China
}

Received March 26, 2017; Accepted October 2, 2017

DOI: $10.3892 / \mathrm{ol} .2017 .7234$

\begin{abstract}
CAMP responsive element binding protein 5 (CREB5) has crucial roles in regulating cell growth, proliferation, differentiation and cell cycle regulation. CREB5 has been identified to be overexpressed in several types of human cancer. However, the expression characteristics of CREB5 in epithelial ovarian cancer remains unknown, and its potential clinical prognostic significance has not yet been elucidated. In the present study, quantitative polymerase chain reaction (qPCR) and western blot analysis were performed to detect CREB5 mRNA and protein expression levels in 10 fresh tissue and cell lines epithelial ovarian cancer. Furthermore, CREB5 expression was analyzed using immunohistochemical analysis in 125 clinicopathologically characterized ovarian cancers: Stage I+II $(n=31)$, stage III $(n=70)$, stage IV $(n=24)$. The patient survival rate was evaluated using Kaplan-Meier analysis. CREB5 was significantly overexpressed at both the mRNA and protein levels in epithelial ovarian cancer cells. There was a significant positive correlation between high CREB5 expression and increasing the International Federation of Gynecology and Obstetrics (FIGO) stage and pelvic lymph node metastasis $(\mathrm{P}<0.05)$. Patients with high CREB5 expression had a shorter overall survival, whereas patients with low CREB5 expression had longer survival. In addition, patients with high CREB5 expression had shorter relapse-free survival whereas patients with low CREB5 expression had longer relapse-free survival. Univariate logistic regression analysis and stepwise multivariate analysis all revealed that the FIGO stage and high CREB5 expression were significant risk factors for epithelial ovarian cancer $(\mathrm{P}<0.001)$, which suggested that
\end{abstract}

Correspondence to: Dr Shuzhong Yao, Department of Obstetrics and Gynecology, The First Affiliated Hospital, Sun Yat-sen University, 58 Zhongshan 2 Road, Guangzhou, Guangdong 510700, P.R. China

E-mail: yszlfy@163.com

${ }^{*}$ Contributed equally

Key words: CAMP responsive element binding protein 5, promotes, tumor cell invasion, epithelial ovarian cancer
CREB5 upregulation may be associated with a poor prognosis; therefore, it may be an independent prognostic indicator of epithelial ovarian cancer and may serve as a tumor-aggressive gene.

\section{Introduction}

Ovarian cancer is one of the most lethal gynecological malignancies (1). In the US, approximately 22,280 new cases are diagnosed every year, and approximately 14,240 women were predicted to have died of ovarian cancer in 2016 (2). Despite the rapid development of surgical and chemotherapeutic techniques, the 5-year survival rate of patients at an advanced stage of ovarian cancer is $<30 \%$ due to drug resistance, relapse, and the lack of effective early screening and diagnostic methods (3). Therefore, determining the molecular mechanism underlying epithelial ovarian cancer is an essential and important topic for cancer treatment.

cAMP response element-binding 5 (CREB5) is a transcriptional factor in eukaryotic cells, and it functions as a protein-coding gene. Its product is a member of the cAMP response element (CRE)-binding protein family. The encoded protein specifically binds to CREasahomodimeroraheterodimer with c-Jun or CRE-BP1, and functions as a CRE-dependent trans-activator $(4,5)$. Members of this family contain zinc-finger and bZIP DNA-binding domains. Few studies have suggested that the up-regulation of CREB5 is negatively correlated with the prognosis of colorectal cancer and non-small cell lung cancer (6,7). However, the relationship between CREB5 and the clinical prognosis of ovarian cancer still needs to be verified. Therefore, here we investigated CREB5 expression and its relationship with clinical prognosis in epithelial ovarian cancer by analyzing its expression level in cancer cell lines and cancer tissues.

\section{Materials and methods}

Cell lines and cell culture. Human ovarian surface epithelial cells (HOSEpiC) (8) and immortalized normal ovarian surface epithelial cells (IOSE80) were purchased from ScienCell Research Laboratories (San Diego, CA, USA) and cultured in ovarian epithelial cell medium. normal ovarian-adjacent tissues were purchased from ScienCell Research Laboratories in 2016 and this cells were grown in 1:1 combination of two 
media, Medium 199 (Invitrogen; Thermo Fisher Scientific, Inc., Waltham, MA, USA) and MCDB 105 (Cell Applications Inc., San Diego, CA, USA) with $10 \%$ FBS in a humidified atmosphere containing $5 \% \mathrm{CO}_{2}$ at $37^{\circ} \mathrm{C}$. Ovarian cancer cell lines including COV362.4, A2780, TOV21G, EFO27, COV644, SKOV3, EFO21, TOV112D, OV90, and OV56 were purchased from the European Collection of Authenticated Cell Cultures (ECACC) in 2016 and cultured in Dulbecco's modified Eagle's medium (Invitrogen; Thermo Fisher Scientific, Inc.), supplemented with $10 \%$ fetal bovine serum (HyClone, Logan, UT, USA).

Patient information and tissue specimens. A total of 125 paraffin-embedded and archived epithelial ovarian cancer samples, which were histopathologically and clinically diagnosed at the Sun Yat-sen University Cancer Center from 2001 to 2006, were analyzed in this retrospective study. All tumors were staged according to the International Federation of Gynaecology and Obstetrics standards (FIGO). Ten freshly collected epithelial ovarian cancer tissues and normal ovarian-adjacent tissues were frozen and stored in liquid nitrogen until further use, including FIGO stage I+II ( $n=31$ ), stage III $(n=70)$, stage IV $(n=24)$. For the use of the clinical materials for research purposes, prior patient consent and approval were obtained from the Institutional Ethical Board (IRB) in the First Affiliated Hospital of Sun Yat-sen University.

\section{Quantitative polymerase chain reaction ( $q P C R)$}

$R N A$ extraction and $q P C R$. Total RNA from cultured cells was extracted from ten fresh ovarian cancer tissue and adjacent normal tissue samples using TRIzol reagent according to the manufacturer's instructions. Complementary DNA (cDNA) was amplified and quantified using an ABI Prism 7500 Sequence Detection system (Applied Biosystems; Thermo Fisher Scientific, Inc.) and SYBR-Green I (Molecular Probes; Invitrogen; Thermo Fisher Scientific, Inc.). qPCR was used to quantify the CREB5 mRNA levels. The RT-PCR conditions for genes were set at $95^{\circ} \mathrm{C}$ for $10 \mathrm{~min}$, followed by 40 cycles at $95^{\circ} \mathrm{C}$ for $20 \mathrm{sec}, 60^{\circ} \mathrm{C}$ for $30 \mathrm{sec}$ and $72^{\circ} \mathrm{C}$ for $1 \mathrm{~min}$. We examined the CREB5 expression by $\mathrm{qPCR}$ and the expression of the mRNA was defined based on $\mathrm{Ct}$, and relative expression levels were calculated as 2-[(Cq of CREB5)-(Cq of GAPDH)] after normalization with reference to the expression of housekeeping gene GAPDH, where $\mathrm{Cq}$ represents quantification cycle. The CREB5 sequences were 5'-AGATGGTCCTCT GTTGGGAA-3' (forward) and 5'-TGGACACGGTTATGA GAATGA-3' (reverse), and those for GAPDH were 5'-AAT GAAGGGGTCATTGATGG-3' (forward) and 5'-AAGGTG AAGGTCGGAGTCAA-3' (reverse).

Western blotting. Cell lysates from cell lines and fresh tissue samples were prepared using cold RIPA buffer. Fresh tissue samples were milled to powder in liquid nitrogen and lysed by SDS-PAGE sample buffer. The protein samples $(30 \mu \mathrm{g})$ were separated on $7.5 \%$ SDS polyacrylamide gels, transferred to polyvinylidene fluoride (PVDF) membranes (Immobilon P; EMD Millipore, Bedford, MA, USA), and blocked with 10\% nonfat dried milk (NFDM) freshly in Tris-buffered saline containing $0.1 \%$ Tween-20 (TBST) for $1 \mathrm{~h}$ at room temperature. After blocking, the membranes were incubated with
anti-CREB5 monoclonal antibodies (1:1,000; GTX44660; BD Biosciences, Franklin Lakes, NJ, USA) overnight at $4^{\circ} \mathrm{C}$. Then, the membranes were rinsed thrice with TBST for $10 \mathrm{~min}$ each time, and incubated with horseradish peroxidase-conjugated goat anti-rabbit IgG (1:2,000; ABIN2474414; Santa Cruz Biotechnology, Inc., Dallas, TX, USA) for $2 \mathrm{~h}$ for $2 \mathrm{~h}$ at room temperature. Then, bound antibodies were detected using an enhanced chemiluminescence detection system (Amersham Pharmacia Biotech, Piscataway, NJ, USA) according to the manufacturer's instructions. GADPH (1:1,000; ab9485; Santa Cruz Biotechnology, Inc.) was chosen as the loading control. The signals of western blotting bands of figures were quantified by densitometry, which determined by comparing the ratio in IOSE80, i.e., the ratio CREB5/GAPDH, in IOSE80 was considered as 1.0 .

Immunohistochemical assay. The CREB5 protein expression levels in the human ovarian cancer tissues were detected by immunohistochemical analysis. Briefly, $4 \mu \mathrm{m}$-thick paraffin-embedded sections were baked at $60^{\circ} \mathrm{C}$ for $1 \mathrm{~h}$, deparaffinized with xylene, rehydrated, and microwaved in EDTA antigen retrieval buffer. Next, high tension was used for antigen retrieval, and the specimens were treated with $3 \%$ hydrogen peroxide in methanol to quench endogenous peroxidase activity, followed by incubation with $1 \%$ bovine serum albumin (36102ES10; Sigma-Aldrich; Merck KGaA, Darmstadt, Germany) to block nonspecific binding for $20 \mathrm{~min}$ at room temperature, and incubation with anti-CREB5 monoclonal antibodies (1:100; ABIN599087; Santa Cruz Biotechnology, Inc.) at $4{ }^{\circ} \mathrm{C}$ overnight. Normal goat serum (Abcam, ab7481) was used as the negative control and we incubate normal goat serum at 4 degrees Celsius overnight. After washing, the tissue sections were treated with biotinylated anti-mouse secondary antibody (1:100; PA128568; Sigma-Aldrich; Merck KGaA), then incubated with streptavidin horseradish peroxidase complex (Sigma-Aldrich; Merck $\mathrm{KGaA}$ ) for $30 \mathrm{~min}$ at $37^{\circ} \mathrm{C}$, immersed in 3-amino-9-ethyl carbazole. the biotinylated anti-mouse secondary antibody and the streptavidin horseradish peroxidase complex are part of the streptavidin-biotin kit and the catalogue number of the streptavidin-biotin kit is SP2002. The sections were then counterstained with 10\% Mayer's hematoxylin, dehydrated, and mounted in Crystal Mount. biotin-labeled anti-mouse IgG goat antibody (dilution 1:100; Sigma-Aldrich; Merck KGaA) as the control $\mathrm{IgG}$. The distribution, positive intensity, and positive ratio of CREB5 were observed by microscopy by two independent pathologists who were blinded to the clinical parameters. The staining results were scored on the basis of the following criteria: i) percentage of positive tumor cells in tumor tissue: 0 (0\%), 1 (1-10\%), 2 (11-50\%), 3 (51-70\%), and 4 (71-100\%); and ii) staining intensity: 0 (none), 1 (weak), 2 (moderate), 3 (strong). The staining index was calculated as the staining intensity score $x$ proportion of positive tumor cells (range, 0-12). A final score of $\geq 5$ was considered high expression, the catalogue number of the streptavidin-biotin kit is SP2002, cells were visualized in an Olympus BX51 fluorescence microscope (Olympus, Tokyo, Japan).

Statistical analysis. All data were statistically analyzed using SPSS 18.0 statistical software. The relationship between 
A

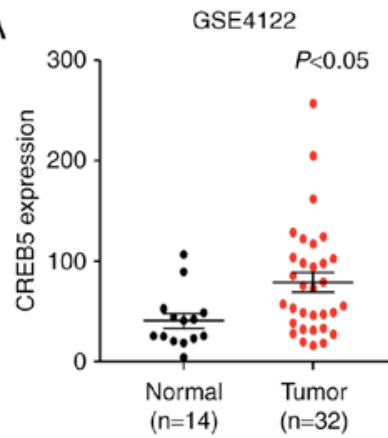

B

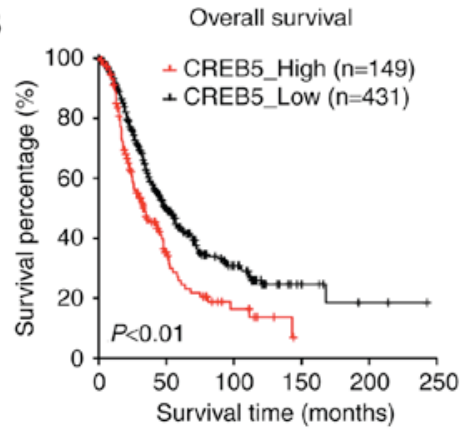

C

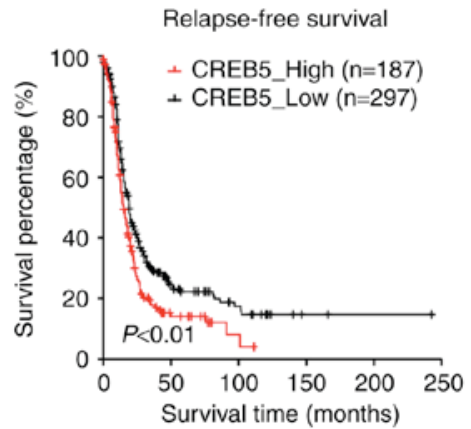

Figure 1. cAMP response element-binding protein 5 (CREB5) is negatively associated with ovarian cancer prognosis. (A) CREB5 expression profiling from GSE41 2 datasets ( $\mathrm{n}=46$ ). (B) Overall survival of patients with low vs. high CREB5 expression in the GSE4122 dataset $(\mathrm{n}=580)$. (C) Relapse-free survival of patients with low vs. high CREB5 expression in the GSE4122 dataset $(n=484)$.

CREB5 expression and clinicopathological characteristics was analyzed using the chi-square test. The Kaplan-Meier method was used to establish survival curves, and the survival differences were compared using the log-rank test. Survival data were evaluated using univariate and multivariate Cox regression analyses. $\mathrm{P}<0.05$ was considered to indicate a statistically significant difference.

\section{Results}

CREB5 expression profile. We analyzed previously published CREB5 expression profiles obtained from 32 patients with epithelial ovarian cancer (GSE4122). The results of our analysis revealed that CREB5 was significantly upregulated in the 32 epithelial ovarian cancer tissues compared to the 14 normal HOSEpiC cell lines analyzed (Fig. 1A). Importantly, in patients of the GSE4122 dataset, higher CREB5 expression was associated with shorter relapse-free survival time and overall survival time, whereas lower CREB5 expression was associated with longer relapse-free survival and overall survival $(\mathrm{P}<0.05$; Fig. 1B and $\mathrm{C})$. In conclusion, these results indicate a possible link between CREB5 overexpression and the progression of human epithelial ovarian cancer.

CREB5 expression in epithelial ovarian cancer cell lines and tissues. CREB5 mRNA and protein expression levels were determined in ten epithelial ovarian cancer cell lines and ten epithelial ovarian cancer samples relative to HOSEpiC and IOSE80 cells were determined using qPCR and western blot analysis. Comparison of the results showed that CREB5 mRNA and protein levels were differentially upregulated in all ten epithelial ovarian cancer samples compared with the normal tissues and cells (Fig. 2). Collectively, the result demonstrated that CREB5 was upregulated in epithelial ovarian cancer.

CREB5 protein expression in epithelial ovarian cancer tissues and association with clinical features. Based on the immunohistochemistry score, we found that CREB5 expression in epithelial ovarian cancer tissues was positively correlated with FIGO stage. Immunohistochemical staining revealed that CREB5 distribution was localized to the nuclei (Fig. 3A). We also explored the relationship between CREB5 and clinical and pathological characteristics and found that CREB5 expression was positively correlated with FIGO stage and pelvic lymph node metastasis $(\mathrm{P}<0.001)$. There was no significant correlation between CREB5 expression and histological type or patient age at surgery ( $\mathrm{P}>0.05$, Table I), suggesting that epithelial ovarian cancer progression was associated with increase in CREB5 expression. We examined the relationship between CREB5 expression and clinical outcomes $(\mathrm{P}<0.05$; Table I). As shown in Fig. 3B and $\mathrm{C}$, patients with high/low CREB5 expression presented different recurrence and survival states. Patients with higher CREB5 expression had significantly longer disease-free survival and overall survival than those with low CREB5 expression. Results of the univariate logistic regression and stepwise multivariate analyses showed that the FIGO stage and high CREB5 expression levels were significant risk factors for epithelial ovarian cancer $(\mathrm{P}<0.001$; Table II). Taken together, our findings suggest that CREB5 may be an independent prognostic biomarker in patients with epithelial ovarian cancer.

\section{Discussion}

Despite several advances in treatment, including chemotherapy and cytoreductive surgery, ovarian cancer remains to be the most lethal malignant gynecological cancer. However, most patients with ovarian cancer that respond to initial therapy subsequently relapse within 5 years (9), and the 5 -year survival rate is approximately $2.4-23 \%(10,11)$. In general, $>50 \%$ of treated patients experience tumor recurrence and ultimately succumb to this malignancy $(12,13)$. Therefore, it is important to identify novel markers in epithelial ovarian cancer, which can advance and initiate more individualized treatment.

In the present study, we first identified the overexpression of CREB5 in cell lines and fresh tissues of epithelial ovarian cancer at both the mRNA and protein levels. Immunostaining analysis revealed a positive correlation between CREB5 expression and FIGO stage. Furthermore, CREB5 overexpression was found to correlates with epithelial ovarian cancer progression, patients with higher CREB5 expression had shorter overall survival time and relapse-free survival time.

Our findings suggest that CREB5 is an independent prognostic indicator of poor outcomes in epithelial ovarian cancer patients. CREB5 is a potential therapeutic target for 
A

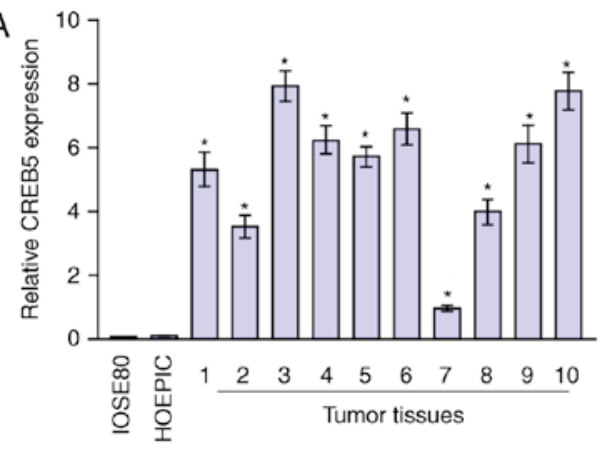

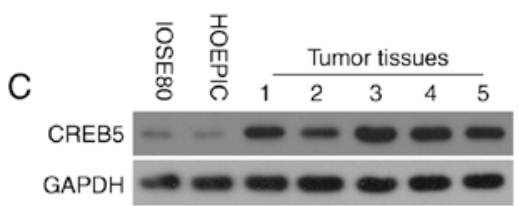

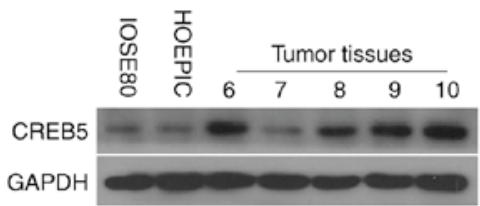

$\mathrm{B}$

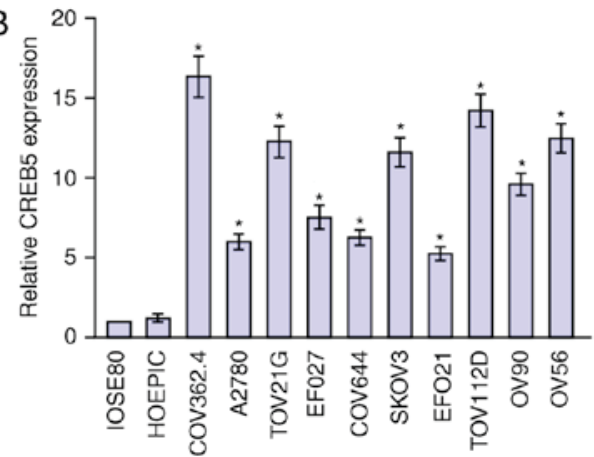

D
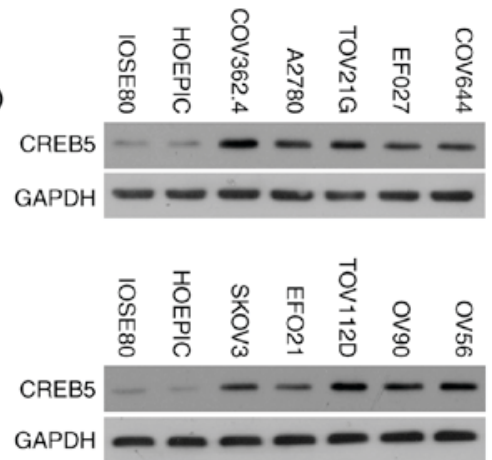

Figure 2. (A and B) qPCR analysis of CREB5 expression in (B) 10 ovarian cancer cell lines and (A) 10 ovarian cancer samples compared to human ovarian surface epithelial cells (HOSEpiC) and IOSE80. Transcript levels were normalized to GAPDH expression. Bars represent the mean \pm standard deviation of three independent experiments. ${ }^{*} \mathrm{P}<0.05$. (C) Western blot analysis of CREB5 protein expression in normal ovarian epithelial cells (HOSEpiC and IOSE80) and primary ovarian tumors from 10 patients with ovarian cancer (T1-T10). GAPDH was used as the loading control. (D) Western blot assay of CREB5 protein expression in normal ovarian epithelial cells and ovarian cancer cell lines. IOSE80 cells were used as the negative control.

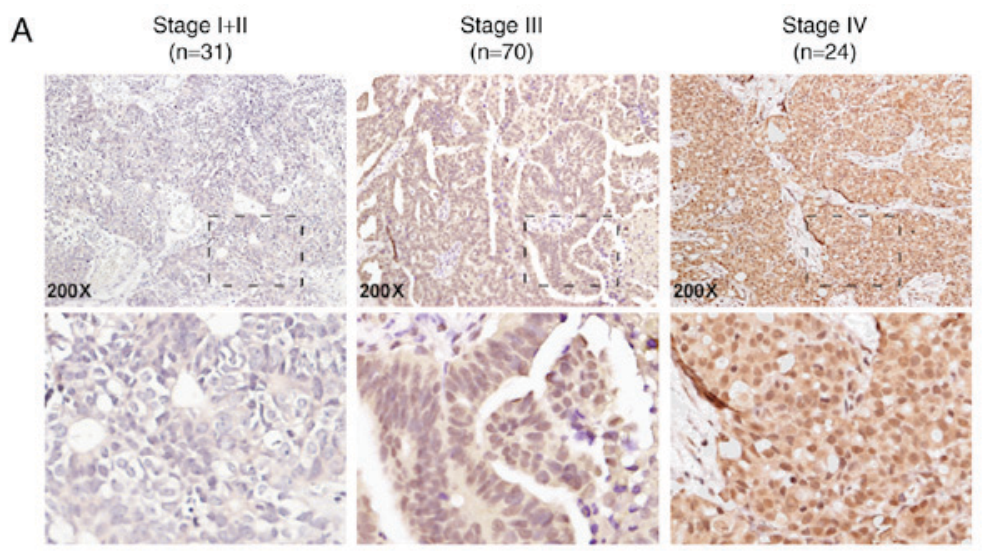

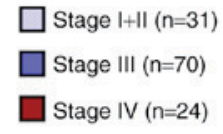

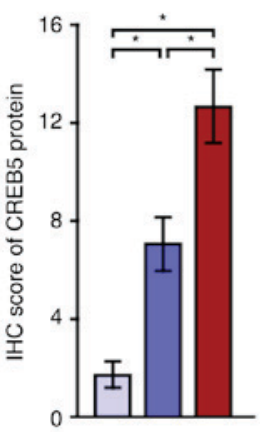

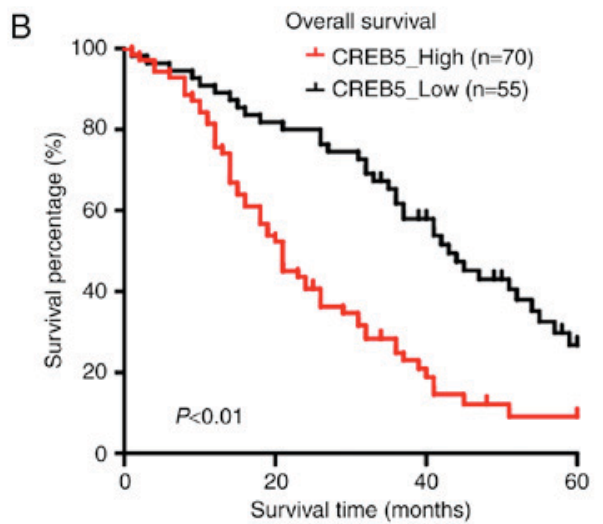

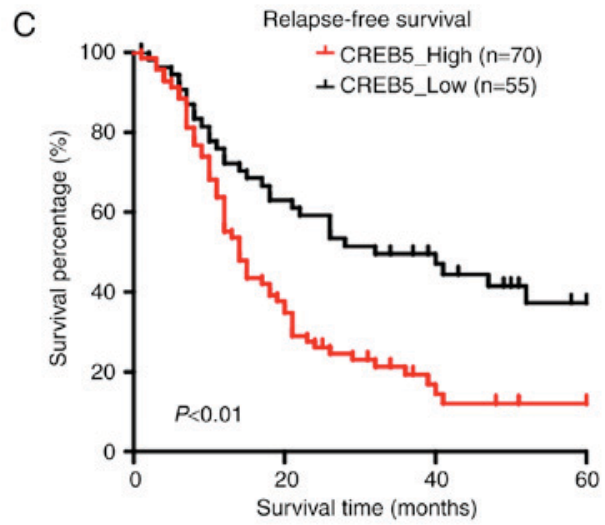

Figure 3. (A) Immunohistochemical staining of CREB5 in human ovarian epithelial cancer tissues. "P<0.05. (B) Overall survival of patients with low vs. high expression of CREB5 in human ovarian surface epithelial cells $(\mathrm{P}<0.01)$. (C) Relapse-free survival (RFS) of patients with low vs. high CREB5 expression in ovarian cancer cells $(\mathrm{P}<0.01)$. 
Table I. The relationship between CREB5 and clinical pathological characteristics in 125 patients with ovarian cancer.

\begin{tabular}{|c|c|c|c|c|}
\hline \multirow[b]{2}{*}{ Parameters } & \multirow[b]{2}{*}{ Number of cases } & \multicolumn{2}{|c|}{ CREB5 expression } & \multirow[b]{2}{*}{ P-values } \\
\hline & & $\operatorname{High}(\mathrm{n}=70)$ & Low $(n=55)$ & \\
\hline \multicolumn{5}{|l|}{ Age (years) } \\
\hline$<55$ & 62 & 40 & 32 & \multirow[t]{2}{*}{0.391} \\
\hline$\geq 55$ & 63 & 30 & 33 & \\
\hline \multicolumn{5}{|l|}{$\begin{array}{l}\text { Histological } \\
\text { type }\end{array}$} \\
\hline Serous & 96 & 58 & 38 & \multirow[t]{5}{*}{0.193} \\
\hline Mucinous & 10 & 4 & 6 & \\
\hline Clear cell & 8 & 6 & 2 & \\
\hline Endometrioid & 9 & 4 & 5 & \\
\hline Others & 2 & 1 & 1 & \\
\hline \multicolumn{5}{|l|}{ FIGO stage } \\
\hline I or II & 31 & 10 & 21 & \multirow[t]{3}{*}{0.001} \\
\hline III & 70 & 40 & 30 & \\
\hline IV & 24 & 20 & 4 & \\
\hline \multicolumn{5}{|l|}{$\begin{array}{l}\text { Pelvic lymph } \\
\text { node metastasis }\end{array}$} \\
\hline Yes & 86 & 56 & 30 & \multirow[t]{2}{*}{0.003} \\
\hline No & 39 & 14 & 25 & \\
\hline \multicolumn{5}{|l|}{ Recurrence } \\
\hline Yes & 88 & 57 & 31 & \multirow[t]{2}{*}{0.003} \\
\hline No & 37 & 13 & 24 & \\
\hline \multicolumn{5}{|l|}{ Survival status } \\
\hline Dead & 94 & 58 & 36 & \\
\hline NO & 31 & 12 & 19 & 0.036 \\
\hline
\end{tabular}

FIGO, International Federation of Gynecology and Obstetrics; CREB5, CAMP responsive element binding protein 5.

Table II. Univariate and multivariate analysis of factors associated with overall survival in 125 ovarian cancer patients.

\begin{tabular}{llrlrr}
\hline & \multicolumn{2}{c}{ Univariate analysis } & & \multicolumn{2}{c}{ Multivariate analysis } \\
\cline { 2 - 3 } Characteristics & HR $(95 \% \mathrm{CI})$ & P-values & & HR $(95 \% \mathrm{CI})$ & P-values \\
\hline Age (years) & $0.993(0.978-1.008)$ & 0.370 & & $0.993(0.979-1.008)$ & 0.373 \\
Histologic grade & $0.860(0.528-1.398)$ & 0.542 & & $0.993(0.609-1.620)$ & 0.978 \\
FIGO stage & $3.961(2.218-7.070)$ & $<0.001$ & & $3.940(2.164-7.175)$ & $<0.001$ \\
CREB5 & $2.629(1.697-4.072)$ & $<0.001$ & & $2.722(1.723-4.299)$ & $<0.001$ \\
\hline
\end{tabular}

HR, hazard ratio; CI, confidence interval; FIGO, International Federation of Gynecology and Obstetrics.

epithelial ovarian cancer. Furthermore, results of the univariate logistic regression and stepwise multivariate analyses revealed that FIGO stage and high CREB5 expression level were significant risk factors for epithelial ovarian cancer ( $\mathrm{P}<0.001$; Table II). Additionally, high CREB5 expression was significantly positively associated with ascending FIGO stage (Table I; Fig. 3). Thus, according to the results of the logistic regression analysis, high CREB5 expression is an independent risk factor for overall survival in patients with epithelial ovarian cancer. CREB is an important transcription factor that regulates diverse cellular processes, including cell differentiation, proliferation, survival, glucose metabolism, immune regulation, and synaptic plasticity associated with memory (14-20).

Similar findings have been reported for other tumors, Qi and Ding analyzed gene expression profiles in colorectal cancer and studied how CREB5 gene expression levels affect the molecular events in colorectal cancer, and found that these 
molecular events were correlated with tumor metastasis (6). Seo et al reported that CREB and phosphorylated CREB mRNA and protein levels were significantly higher in most non-small cell lung cancer cell lines and tumor specimens than in the normal human tracheobronchial epithelial cells and adjacent normal lung tissue, respectively (7). Analysis of CREB mRNA expression and gene copy number showed that CREB overexpression occurred mainly at the transcriptional level.

CREB could activate the transcription of downstream genes by binding to CRE. Alternatively spliced transcript variants encoding different isoforms have been identified. Gene ontology annotations related to this gene include nucleic acid binding and sequence-specific DNA binding. The study of Seo et al found that CREB expression levels in non-small cell lung cancer cells were highly dependent on the elevated CREB activity for their survival and cell growth, and inhibition of CREB effectively suppressed the growth of non-small cell lung cancer cells (7). Kinjo et al reported that a majority of patients with acute lymphoid and myeloid leukemia overexpressed CREB in the bone marrow. CREB overexpression was associated with poor initial outcome of clinical disease in patients with acute myelocytic leukemia (21). Our study proved that CREB5 was significantly overexpressed in epithelial ovarian cancer. The CREB5 expression level was significantly correlated with aggressive features and an unfavorable prognosis in 125 patients with ovarian cancer. Furthermore, Kaplan-Meier curves and multivariate Cox proportional hazard regression analysis indicated that CREB5 overexpression was a strong and independent predictor for poor overall survival in patients with ovarian carcinoma. These findings revealed that CREB5 might play an important role as an indicator of poor outcomes in individual patients with ovarian cancer.

In conclusion, our study revealed that CREB5 played an important role in the development and progression of epithelial ovarian cancer, and CREB5 overexpression was positively associated with the ascending FIGO stage and poor survival of epithelial ovarian cancer. Therefore, we believe that CREB5 may be a potential target for epithelial ovarian cancer therapy. The results showed in this manuscript were preliminary data. In future studies, samples should be enriched, because the present study analyzed only 125 epithelial ovarian cancer cases. We should further verify the role and function of CREB5 in ovarian cell lines. The gain- and loss-of function of CREB5 in ovarian cell lines should be performed. The abilities of growth and motility in gain- and loss-function of CREB5 in ovarian cell lines should be tested. These results may provide a strong evidence to support the clinical data.

\section{Acknowledgements}

This study was supported by grants from the Science and Technology Planning Project of Guangdong Province, China (grand no. 2016A030313820); the Science and Technology
Planning Project of Huangpu Guangdong Province, China (grand no. 201544-03); and the Science and Technology Planning Project of Guangzhou City, China (grand no. 201704020163).

\section{References}

1. Gloss BS and Samimi G: Epigenetic biomarkers in epithelial ovarian cancer. Cancer Lett 342: 257-263, 2014.

2. Matulonis UA, Sood AK, Fallowfield L, Howitt BE, Sehouli J and Karlan BY: Ovarian cancer. Nat Rev Dis Primers 2: 16061, 2016.

3. Aletti GD, Gallenberg MM, Cliby WA, Jatoi A and Hartmann LC: Current management strategies for ovarian cancer. Mayo Clin Proc 82: 751-770, 2007.

4. Zhang X, Liu H, Xie Z, Deng W, Wu C, Qin B, Hou J and Lu M: Epigenetically regulated miR-449a enhances hepatitis B virus replication by targeting cAMP-responsive element binding protein 5 and modulating hepatocytes phenotype. Sci Rep 6: 25389, 2016.

5. Zu YL, Maekawa T, Nomura N, Nakata T and Ishii S: Regulation of trans-activating capacity of CRE-BPa by phorbol ester tumor promoter TPA. Oncogene 8: 2749-2758 1993.

6. Qi L and Ding Y: Involvement of the CREB5 regulatory network in colorectal cancer metastasis. Yi Chuan 36: 679-684, 2014.

7. Seo HS, Liu DD, Bekele BN, Kim MK, Pisters K, Lippman SM, Wistuba II and Koo JS: Cyclic AMP response element-binding protein overexpression: A feature associated with negative prognosis in never smokers with Non-small cell lung cancer. Cancer Res 68: 6065-6073, 2008

8. Jarboe EA, Folkins AK, Drapkin R, Ince TA, Agoston ES and Crum CP: Tubal and ovarian pathways to pelvic epithelial cancer: A pathological perspective. Histopathology 53: 127-138, 2008.

9. Hennessy BT, Coleman RL and Markman M: Ovarian cancer. Lancet 374: 137-1382, 2009.

10. Jemal A, Bray F, Center MM, Ferlay J, Ward E and Forman D: Global cancer statistics. CA Cancer J Clin 61: 69-90, 2011.

11. Grann AF, Nørgaard M, Blaakær J, Søgaard-Andersen E and Jacobsen JB: Survival of patients with ovarian cancer in central and northern Denmark, 1998-2009. Clin Epidemiol 3 (Suppl 1): S59-S64, 2011.

12. Bender E: Trials show delayed recurrence in ovarian cancer. Cancer Discov 3: OF8, 2013.

13. Amate P, Huchon C, Dessapt AL, Bensaid C, Medioni J, Le Frère Belda MA, Bats AS and Lécuru FR: Ovarian cancer: Sites of recurrence. Int J Gynecol Cancer 23: 1590-1596, 2013.

14. Wen AY, Sakamoto KM and Miller LS: The role of the transcription factor CREB in immune function. J Immunol 185: 6413-6419, 2010.

15. Carlezon WA Jr, Duman RS and Nestler EJ: The many faces of CREB. Trends Neurosci 28: 436-445, 2005.

16. Silva AJ, Kogan JH, Frankland PW and Kida S: CREB and memory. Annu Rev Neurosci 21: 127-148, 1998.

17. Mayr B and Montminy M: Transcriptional regulation by the phosphorylation-dependent factor CREB. Nat Rev Mol Cell Biol 2: 599-609, 2001.

18. Arnould T, Vankoningsloo S, Renard P, Houbion A, Ninane N Demazy C, Remacle J and Raes M: CREB activation induced by mitochondrial dysfunction is a new signaling pathway that Impairs cell proliferation. EMBO J 21: 53-63, 2002.

19. Vaudry D, Stork PJ, Lazarovici P and Eiden LE: Signaling pathways for PC12 cell differentiation: Making the right connections. Science 296: 1648-1649, 2002.

20. Bender RA, Lauterborn JC, Gall CM, Cariaga W and Baram TZ: Enhanced CREB phosphorylation in immature dentate gyrus granule cells precedes neurotrophin expression and indicates a specific role of CREB in granule cell differentiation. Eur J Neurosci 13: 679-686, 2001.

21. Kinjo K, Sandoval S, Sakamoto KM and Shankar DB: The role of CREB as a proto-oncogene in hematopoiesis. Cell Cycle 4: 1134-1135, 2005. 